\title{
Comparative Efficacy of Herbicides Applied in Wheat and their Residual Effect on the Succeeding Crops
}

\author{
H.L. Yadav ${ }^{1 *}$, A.K. Gupta ${ }^{2}$, Sudesh Kumar ${ }^{1}$ and Shweta Gupta ${ }^{1}$ \\ ${ }^{1}$ Division of Agronomy, Rajasthan Agriculture Research Institute, \\ Durgapura, Jaipur, India \\ ${ }^{2}$ Division of Agronomy, SKNAU, Jobner, Jaipur, Rajasthan, India \\ *Corresponding author
}

\section{A B S T R A C T}

\section{Keywords}

Weed density, Herbicide mixture, Crop productivity, Wheat,

Germination, Succeeding crops

Article Info

Accepted:

15 March 2019

Available Online:

10 April 2019
The field experiment conducted at research farm, RARI, Durgapura for two consecutive years during rabi seasons 2013-14 and 2014-15. Results revealed that maximum reduction in weed density at 25 DAS was recorded with pendimethalin pre emergence @ 0.750 $\mathrm{kg} / \mathrm{ha}$ and at 50 DAS with 2, 4-D ester @ $0.5 \mathrm{~kg} / \mathrm{ha}$, clodinafop propanyl $15 \%+$ metsulfuran methyl 1\% @ $64 \mathrm{~g}$ a.i./ha and metsulfuran methyl @ $4 \mathrm{~g}$ a.i./ha. All the weed control treatments produced significantly higher grain and straw yield compared to weedy check. Hand weeding, except weed free produced the maximum grain and straw yield of 46.40 and $56.20 \mathrm{q} / \mathrm{ha}$ and thus out yielded over rest of the treatment. Being at par with clodinafop propargyl $15 \%+$ metsulfuran methyl $1 \%$ @ $64 \mathrm{~g}$ a.i. /ha and sulfosulfuran 75 $\%$ +metsulfuran methyl 5 WG @ $32 \mathrm{~g}$ a.i. /ha, application of clodinafop propargyl $15 \%+$ metsulfuran methyl 1\% @ $64 \mathrm{~g}$ a.i. /ha registered an increase of 32.6 and 35.1 per cent in grain and straw yield, respectively over weedy check and thus found as the next superior herbicidal treatment. Further, none of the applied herbicides/mixtures in rabi season (wheat) had residual toxicity on germination of predominant crops (pearlmillet, mungbean and clusterbean) grown in kharif season.

\section{Introduction}

Weeds are known to be a major biotic constraint in agricultural production systems. Estimates reveal that more than one-third of the total field losses due to biotic stresses are caused by weeds alone which often go unnoticed due to their multipronged hidden effects on plant growth (Sharma et al., 2016). Weeds have better adaptability to the changing environments by virtue of greater genetic diversity in comparison to crops. Weed management is likely to become more complex in future due to increase in their invasiveness, weed shifts, herbicides resistance in weeds and their residue hazards under changing climate (Kumar et al., 2016). Increasing interest towards conservation agricultural and shortage of labour due to implementation of different development 
programme in the country are increasing the acceptance of herbicides to tackle the dynamic problem of weed management. Herbicides play an important role for weed control in close spaced crops wheat, where manual or mechanical weeding is difficult. Also the mimicry weeds can hardly be weeded out by hand weeding or other mechanical methods. Chemical weed control is most suitable option to overcome this problem.

\section{Materials and Methods}

The field experiment conducted at research farm, RARI, Durgapura for two consecutive years during rabi seasons 2013-14 and 201415 on loamy sand soil. The experiment comprised of eleven weed control treatments i.e. Weedy check $\left(T_{1}\right)$, Hand weeding at 3035 DAS $\left(\mathrm{T}_{2}\right), 2,4-\mathrm{D}$ ester @ $0.5 \mathrm{~kg} / \mathrm{ha}$ at 30 35 DAS $\left(\mathrm{T}_{3}\right)$, Sulfosulfuron @ $25 \mathrm{~g}$ a. i. at 3035 DAS $\left(\mathrm{T}_{4}\right)$, Metsulfuron methyl @ $4 \mathrm{~g} / \mathrm{ha}$ at 30-35 DAS $\left(\mathrm{T}_{5}\right)$, Sulfosulfuron $75 \%+$ Metsulfuron methyl 5\% WG @ 32g at 30-35 DAS $\left(\mathrm{T}_{6}\right)$, Piroxofop- propargyl 15\% WP @ 60g a. i./ha 30-35 DAS ( $\left.\mathrm{T}_{7}\right)$, Clodinafoppropargyl15\% + Metsulfuron methyl 1\% @ 64g a.i./ha at 30-35 DAS ( $\left.\mathrm{T}_{8}\right)$, Carfentrazone ethyl @15 g/ha at 30-35 DAS $\left(\mathrm{T}_{9}\right)$, Pendimethalin pre emergence $\left(\mathrm{T}_{10}\right)$ and Weed free $\left(\mathrm{T}_{11}\right)$ were laid out in randomized block design and replicated four times. Weed density of weed species was taken at 25 and 50 DAS from five random spots in each plot by counting the number of weeds per quadrate of $0.25 \mathrm{~m}^{2}$ and the average was computed. Weed density is also known as absolute density of a species. In order to draw valid conclusion, the weed count data were subjected to square root transformation $(\sqrt{(x+0.5)})$ as suggested by (Gomez and Gomez, 1984) before subjecting to statistical analysis. After complete sun drying, the produce of each net plot was threshed and after proper cleaning, it was weighed and converted into quintals per hectare. The straw yield was calculated by subtracting the corresponding grain yield from the biological yield and then converted into quintals per hectare. To see the residual effect of herbicide on next crop, the counted seeds of pearl millet crop were sown just after harvesting of wheat crop. Germinating plants were counted in each row after 15 days of sowing and germination percentage was calculated by the following formula:-

Germination percentage $(\%)=$

Seeds germinated

X 100

Seeds sown

\section{Results and Discussion}

\section{Effect of different weed control practices on weed count}

A perusal of data presented in Table 1 revealed that pre emergence application of pendimethalin reduced the weed count significantly at this stage of crop growth in comparison to weedy check and other treatments (as they were not applied before 25 days) during both the years as well as in pooled analysis. Pooled results indicated that pendimethalin pre emergence @ $0.750 \mathrm{~kg} / \mathrm{ha}$ were found the most superior treatment that recorded significantly lower weed count of 2.72 per $\mathrm{m}^{2}$ at $25 \mathrm{DAS}$, respectively than rest of the treatments except weed free. In this way, this treatment reduced the weed count to the extent of 92.5 per cent in comparison to weedy check. The data presented in Table 1 further showed that the number of monocot weeds per $\mathrm{m}^{2}$ at 50 DAS was significantly influenced due to various treatments tried in the present experiment. Significantly less number of monocot weeds $\left(5.38 \mathrm{~m}^{2}\right)$ was recorded under treatment hand weeding $(\mathrm{HW})$ plots. Hand weeding reduced the weed count 
to the extent of 86.1 per cent in comparison to weedy check. Among difference post emergence herbicides, mixtures proved superior to individual molecules e.g. T8 and T6.

\section{Dicot weeds}

An appraisal of data (Table 2) revealed that pre emergence application of pendimethalin reduced the weed count significantly at this stage of crop growth in comparison to weedy check during both the years as well as in pooled analysis. Pooled results indicated that dicot weeds per $\mathrm{m}^{2}$ at 25 DAS significantly differed due to different weed control treatments. Among the different treatments tried, except weed free treatment $(0.00)$, the second best treatment emerged out from the study was pendimethalin pre emergence @ $0.750 \mathrm{~kg} / \mathrm{ha}$ where significantly least number of dicot weed (6.14) was observed. This treatment reduced the weed count to the extent of 93.04 per cent, in comparison to weedy check. Perusal of data (Table 2) further indicated that all the weed control treatments either mechanical or herbicidal reduced the weed count at this stage of crop growth in comparison to weedy check during both the years as well as in pooled analysis.

Pooled data indicated that hand weeding was proved most superior which was statistically at par with post emergence application of clodinofop propargyl $15 \%+$ metsulfuran methyl1\%@64 g a.i./ha and 2,4-D ester @ $0.5 \mathrm{~kg}$ a.i./ha. Polled weed count recorded under these treatment were 4.51, 5.81, 5.97 and 6.93 per cent, respectively and reduction in dicot weed count due to these treatments were $89.06,85.90,85.51$ and 83.18 per cent, respectively compared to weed check.. Heavy infestation of weeds under weedy check has also been reported by Agarwal and Jain (1998), Sardana et al., (2001) and Singh and Singh (2005).

\section{Effect of different weed control practices on crop productivity}

Except piroxofop-propargyl $15 \%$ WP 60 g a.i./ha all the weed control treatments significantly improved the grain yield of wheat in comparison to weedy check during both the years as well as in pooled analysis (Table 3). Pooled results showed that weed free produced the maximum grain yield of $47.72 \mathrm{q} / \mathrm{ha}$ which was statistically at par with grain yield under sulfosulfuran $75 \%+$ metsulfuran methyl 5\% WG @ $32 \mathrm{~g}$ a.i./ha hand weeding and clodinafoppropargyl $15 \%$ + metsulfuran methyl 1\% @64 g a.i./ha and significatlly superior over rest of the treatments. It registered remarkable increase in grain yield to the extent of $2.8,4.7,6.4$, $10.7,12.3,15.9,19.3,23.2,27.9$ and 38.9 per cent over hand weeding, clodinafoppropargyl $15 \%$ +metsulfuran methyl 1\% @ $64 \mathrm{~g}$ a.i./ha, sulfosulfuran $75 \%$ +metsulfuran methyl 5\%WG@ 32 g a.i./ha, carfentrazone ethyl $40 \%$ DF @ $20 \mathrm{~g}$ a.i./ha, metsulfuran methyl @ 4 g a.i./ ha, 2,4-D ester @ 0.5 kg/ha, sulfosulfuran @ 25 gm a.i./ha, pendimethalin pre emergence, piroxofoppropargyl $15 \%$ WP $60 \mathrm{~g}$ a.i./ha and weedy check treatments, respectively. Application of hand weeding was found to next best treatment. It provided the grain yield of 4640 $\mathrm{kg} / \mathrm{ha}$ that was $7.7,9.612 .7,16.0,19.8,24.3$ and 35.11 per cent more than carfentrazone ethyl 40 \% DF @ 20 g a.i./ha, metsulfuran methyl @ 4 g a.i./ ha, 2,4-D ester @ 0.5 kg/ha, sulfosulfuran @ 25 gm a.i./ha, pendimethalin pre emergence, piroxofoppropargyl $15 \%$ WP $60 \mathrm{~g}$ a.i./ha and weedy check treatments, respectively. However, it was found at par with clodinafoppropargyl 15 $\%$ +metsulfuran methyl $1 \%$ @ $64 \mathrm{~g}$ a.i./ha (4555 kg /ha) and sulfosulfuran $75 \%$ +metsulfuran methyl 5\% WG @ $32 \mathrm{~g}$ a.i./ha $(44.85 \mathrm{~kg} / \mathrm{ha})$. The corresponding increase in grain yield due to clodinafoppropargyl $15 \%$ +metsulfuran methyl 1\% @ $64 \mathrm{~g}$ a.i./ha and 
sulfosulfuran $75 \%$ +metsulfuran methyl 5\% WG @ $32 \mathrm{~g}$ a.i./ha was 5.7 and 4.1 per cent over carfentrazone ethyl 40\% DF @ 20 g a.i./ha, 7.2 and 5.5 per cent over metsulfuran methyl @ $4 \mathrm{~g}$ a.i./ ha, 10.6 and 8.9 per cent over 2,4-D ester @ $0.5 \mathrm{~kg} / \mathrm{ha}, 13.8$ and 12.1 per cent over sulfosulfuran @ 25 gm a.i./ha, 17.6 and 15.8 per cent over pendimethalin pre emergence, 22.1 and 20.2 per cent over piroxofop-propargyl $15 \%$ WP $60 \mathrm{~g}$ a.i./ha and 32.6 and 30.6 weedy check treatments. carfentrazone ethyl 40\% DF @ $20 \mathrm{~g}$ a.i./ha, metsulfuran methyl @ $4 \mathrm{~g}$ a.i./ ha, 2,4-D ester @ $0.5 \mathrm{~kg} / \mathrm{ha}$, sulfosulfuran@ $25 \mathrm{gm}$ a.i./ha, pendimethalin pre emergence and piroxofoppropargyl $15 \%$ WP $60 \mathrm{~g}$ a.i./ha also gave 25.4, 23.7, 19.8, 16.4, 12.7 and 8.6 per cent more grain yield than weedy check but they were noted less effective than above described treatments. Similar findings were also reported by Nadeem et al., (2007) and Surin et al., (2013) and Kumar et al., (2010).

A critical examination of the data presented in Table 3 revealed that straw yield of wheat was also influenced in same manner due to different weed control treatments as in the grain yield. Pooled results showed that weed free produced the maximum straw yield (5729 $\mathrm{kg} / \mathrm{ha}$ ) which was higher of $1.09,1.83,2.77$, $6.22,6.68,8.37,8.73,9.80,12.31$ and 16.23 q/ha over hand weeding, clodinafoppropargyl $15 \%+$ metsulfuran methyl $1 \%$ @ $64 \mathrm{~g}$ a.i./ha, sulfosulfuran $75 \%$ +metsulfuran methyl 5\%WG @ $32 \mathrm{~g}$ a.i./ha, carfentrazone ethyl 40\% DF @ $20 \mathrm{~g}$ a.i./ha, metsulfuran methyl @ $4 \mathrm{~g}$ a.i./ ha, 2,4-D ester @ 0.5 $\mathrm{kg} / \mathrm{ha}$, sulfosulfuran @ $25 \mathrm{gm}$ a.i./ha, pendimethalin pre emergence, piroxofoppropargyl $15 \%$ WP $60 \mathrm{~g}$ a.i./ha and weedy check treatments, respectively. Application of hand weeding, clodinafoppropargyl $15 \%+$ metsulfuran methyl1\%@64 g a.i./ha and sulfosulfuran $75 \%$ +metsulfuran methyl 5\% WG @ $32 \mathrm{~g}$ a.i./ha were found the next superior and equally effective treatments in enhancing straw yield of wheat. These treatments improved the straw yield to the extent of $36.8,35.0$ and 32.0 per cent over control. Carfentrazone ethyl $40 \%$ DF @ 20 g a.i./ha, metsulfuran methyl @ $4 \mathrm{~g}$ a.i./ ha, 2,4D ester @ $0.5 \mathrm{~kg} / \mathrm{ha}$, sulfosulfuran @ $25 \mathrm{gm}$ a.i./ha, pendimethalin pre emergence and piroxofop-propargyl $15 \% \mathrm{WP} 60 \mathrm{~g}$ a.i./ha also enhanced the straw yield by margin of 24.3, 23.2, 19.1, 18.2, 15.6 and 9.5 per cent. Similar findings were also reported by Nadeem et al., (2007) and Surin et al., (2013).

\section{Residual effect of different weed control practices on germination of various succeeding crops}

\section{Pearl millet}

A perusal of pooled data of two years (Table 4) revealed that highest germination per cent (98\%) was reported in plots that were weed free plot, hand weeded and receiving clodinafop propargyl $15 \%+$ metsulfuran methyl1\% @64 g a.i./ha in previous season followed by 2,4-D ester @ $0.5 \mathrm{~kg} / \mathrm{ha}$, sulfosulfuran @ $25 \mathrm{gm}$ a.i./ha and metsulfuran methyl@4 g a.i./ ha. These treatments attained 97 per cent germination. However, the lowest germination per cent was observed in plots of weedy check plots i.e. 94 per cent.

\section{Mungbean}

A perusal of pooled data of two years (Table 4) revealed that highest germination per cent (97\%) was reported for 2,4-D ester @ 0.5 $\mathrm{kg} / \mathrm{ha}$, sulfosulfuran @ $25 \mathrm{gm}$ a.i./ha, metsulfuran methyl @ $4 \mathrm{~g}$ a.i./ ha, carfentrazone Ethyl 40 \% DF @ 20 g a.i./ha and pendimethalin pre emergence followed by hand weeding, clodinafop propargyl $15 \%$ +metsulfuran methyl $1 \%$ @ $64 \mathrm{~g}$ a.i./ha and sulfosulfuran $75 \%$ +metsulfuran methyl 5\% WG@ 32 g a.i./ha. 
Table.1 Effect of weed control treatments on monocot weed population at 25 and 50 DAS of wheat

\begin{tabular}{|c|c|c|c|c|c|c|}
\hline \multirow[t]{3}{*}{ Treatments } & \multicolumn{6}{|c|}{ Monocot weed population (No $\mathrm{m}^{-2}$ ) } \\
\hline & \multicolumn{3}{|c|}{25 DAS } & \multicolumn{3}{|c|}{50 DAS } \\
\hline & 2014 & 2015 & Pooled & 2014 & 2015 & Pooled \\
\hline \multirow{2}{*}{ Weedy check } & 36.19 & 36.39 & 36.29 & 38.76 & 38.81 & 38.79 \\
\hline & $(6.06)$ & $(6.07)$ & $(6.07)$ & $(6.27)$ & $(6.27)$ & $(6.27)$ \\
\hline \multirow[t]{2}{*}{ Hand weeding at $30-35$ DAS } & 35.67 & 36.15 & 35.91 & 5.37 & 5.39 & 5.38 \\
\hline & $(6.01)$ & $(6.05)$ & $(6.03)$ & $(2.42)$ & $(2.43)$ & $(2.42)$ \\
\hline \multirow[t]{2}{*}{ 2,4-D ester @0.5 kg/ha at 30 - 35 DAS } & 29.82 & 30.22 & 30.02 & 32.07 & 33.17 & 32.62 \\
\hline & $(5.51)$ & $(5.54)$ & $(5.52)$ & $(5.71)$ & $(5.8)$ & $(5.75)$ \\
\hline \multirow[t]{2}{*}{ Sulfosulfuran@ 25 gm a.i./ha at 30 - 35 DAS } & 30.78 & 30.79 & 30.79 & 10.37 & 10.75 & 10.56 \\
\hline & $(5.59)$ & $(5.59)$ & $(5.59)$ & $(3.3)$ & $(3.35)$ & $(3.33)$ \\
\hline \multirow[t]{2}{*}{ Metsulfuran Methyl @ 4 g a.i. / ha at 30-35 DAS } & 30.67 & 31.17 & 30.92 & 31.67 & 32.62 & 32.15 \\
\hline & $(5.58)$ & $(5.63)$ & $(5.61)$ & $(5.67)$ & $(5.75)$ & $(5.71)$ \\
\hline \multirow[t]{2}{*}{ Sulfosulfuran $75 \%$ +metsulfuran methyl 5\% WG @ $32 \mathrm{~g}$ a.i. /ha at 30 - 35 DAS } & 31.87 & 32.88 & 32.38 & 9.11 & 9.59 & 9.35 \\
\hline & $(5.69)$ & (5.78) & $(5.73)$ & $(3.1)$ & $(3.18)$ & $(3.14)$ \\
\hline \multirow[t]{2}{*}{ Piroxofop-Propargyl $15 \%$ WP $60 \mathrm{~g}$ a.i. /ha at 30 - 35 DAS } & 33.67 & 34.07 & 33.87 & 11.92 & 12.1 & 12.01 \\
\hline & $(5.85)$ & (5.88) & $(5.86)$ & $(3.52)$ & $(3.55)$ & $(3.54)$ \\
\hline \multirow[t]{2}{*}{ Clodinafoppropargyl15 \% +metsulfuran methyl $1 \%$ @ 64 g a.i. /ha at 30 - 35 DAS } & 33.09 & 34.19 & 33.64 & 7.92 & 7.99 & 7.96 \\
\hline & $(5.8)$ & $(5.89)$ & $(5.84)$ & $(2.9)$ & $(2.91)$ & $(2.91)$ \\
\hline \multirow[t]{2}{*}{ Carfentrazone Ethyl 40 \% DF @ 20 g a.i./ha at 30 - 35 DAS } & 30.9 & 30.00 & 30.45 & 33.9 & 34 & 34.03 \\
\hline & $(5.6)$ & $(5.52)$ & $(5.56)$ & $(5.87)$ & $(5.89)$ & $(5.88)$ \\
\hline \multirow[t]{2}{*}{ Pendimethalin pre emergence } & 2.68 & 2.75 & 2.72 & 22.03 & 22.23 & 22.13 \\
\hline & $(1.78)$ & $(1.80)$ & (1.79) & $(4.75)$ & $(4.77)$ & $(4.76)$ \\
\hline \multirow[t]{2}{*}{ Weed free } & 0 & 0 & 0 & 0 & 0 & 0 \\
\hline & $(0.71)$ & $(0.71)$ & $(0.71)$ & $(0.71)$ & $(0.71)$ & $(0.71)$ \\
\hline SEm \pm & 1.11 & 1.31 & 0.86 & 0.88 & 0.81 & 0.60 \\
\hline $\mathrm{CD}(\mathrm{P}=\mathbf{0 . 0 5})$ & 3.20 & 3.79 & 2.43 & 2.53 & 2.33 & 1.68 \\
\hline
\end{tabular}


Table.2 Effect of weed control treatments on dicot weed population at 25 and 50 DAS of wheat

\begin{tabular}{|c|c|c|c|c|c|c|}
\hline \multirow[t]{3}{*}{ Treatments } & \multicolumn{6}{|c|}{ Dicot Weed population $\left(\mathrm{No}^{-2}\right.$ ) } \\
\hline & \multicolumn{3}{|c|}{25 DAS } & \multicolumn{3}{|c|}{50 DAS } \\
\hline & 2014 & 2015 & Pooled & 2014 & 2015 & Pooled \\
\hline \multirow[t]{2}{*}{ Weedy check } & 36.13 & 38.03 & 37.08 & 41.13 & 41.28 & 41.21 \\
\hline & $(6.3)$ & $(6.37)$ & $(6.33)$ & $(6.45)$ & $(6.46)$ & $(6.46)$ \\
\hline \multirow[t]{2}{*}{ Hand weeding at 30 - 35 DAS } & 35.18 & 34.82 & 35.00 & 4.36 & 4.66 & 4.51 \\
\hline & $(5.97)$ & $(5.94)$ & $(5.96)$ & $(2.20)$ & $(2.27)$ & $(2.24)$ \\
\hline \multirow[t]{2}{*}{ 2,4-D ester @ $0.5 \mathrm{~kg} / \mathrm{ha}$ at 30 - 35 DAS } & 34.78 & 36.43 & 35.61 & 6.80 & 7.05 & 6.93 \\
\hline & $(5.94)$ & $(6.08)$ & $(6.01)$ & $(2.70)$ & $(2.75)$ & $(2.72)$ \\
\hline \multirow[t]{2}{*}{ Sulfosulfuran@25 gm a.i./ha at 30 - 35 DAS } & 35.16 & 37.00 & 36.08 & 36.7 & 37.17 & 36.94 \\
\hline & $(5.97)$ & $(6.12)$ & $(6.05)$ & $(6.10)$ & $(6.14)$ & $(6.12)$ \\
\hline \multirow[t]{2}{*}{ Metsulfuran Methyl @ 4 g a.i. / ha at 30-35 DAS } & 31.92 & 33.17 & 32.55 & 5.93 & 6.00 & 5.97 \\
\hline & $(5.69)$ & $(5.8)$ & $(5.75)$ & $(2.54)$ & $(2.55)$ & $(2.54)$ \\
\hline \multirow[t]{2}{*}{ Sulfosulfuran $75 \%$ +metsulfuran methyl 5\% WG @ 32 g a.i. /ha at 30 - 35 DAS } & 35.04 & 36.92 & 35.98 & 7.93 & 8.13 & 8.03 \\
\hline & $(5.96)$ & (6.12) & (6.04) & $(2.90)$ & $(2.94)$ & $(2.92)$ \\
\hline \multirow[t]{2}{*}{ Piroxofop-Propargyl $15 \%$ WP 60 g a.i. /ha at 30 - 35 DAS } & 35.16 & 35.11 & 35.14 & 36.78 & 36.88 & 36.83 \\
\hline & $(5.97)$ & $(5.97)$ & $(5.97)$ & (6.11) & (6.11) & $(6.11)$ \\
\hline \multirow[t]{2}{*}{ Clodinafop-propargyl15 \% +metsulfuran methyl $1 \%$ @ 64 g a.i. /ha at 30 - 35 DAS } & 34.92 & 35.15 & 35.04 & 5.73 & 5.89 & 5.81 \\
\hline & $(5.95)$ & $(5.97)$ & $(5.96)$ & $(2.50)$ & $(2.53)$ & $(2.51)$ \\
\hline \multirow[t]{2}{*}{ Carfentrazone Ethyl 40 \% DF @ 20 g a.i./ha at 30 - 35 DAS } & 32.67 & 33.00 & 32.92 & 6.87 & 7.00 & 6.93 \\
\hline & $(5.76)$ & $(5.8)$ & $(5.78)$ & $(2.71)$ & $(2.74)$ & $(2.73)$ \\
\hline \multirow[t]{2}{*}{ Pendimethalin pre emergence } & 6.16 & $6.12)$ & 6.14 & 25.43 & 25.81 & 25.62 \\
\hline & $(2.58)$ & $(2.57)$ & $(2.58)$ & (5.09) & $(5.13)$ & $(5.11)$ \\
\hline \multirow[t]{2}{*}{ Weed free } & 0 & 0 & 0 & 0 & 0 & 0 \\
\hline & $(0.71)$ & $(0.71)$ & $(0.71)$ & $(0.71)$ & $(0.71)$ & $(0.71)$ \\
\hline SEm \pm & 1.46 & 1.49 & 1.04 & 0.67 & 0.89 & 0.56 \\
\hline $\mathrm{CD}(\bar{P}=0.05)$ & 4.21 & 4.29 & 2.94 & 1.94 & 2.58 & 1.58 \\
\hline
\end{tabular}


Table.3 Effect of weed control treatments on grain and straw yield of wheat

\begin{tabular}{|c|c|c|c|c|c|c|}
\hline \multirow[t]{2}{*}{ Treatments } & \multicolumn{3}{|c|}{ Grain yield (q/ha) } & \multicolumn{3}{|c|}{ Straw yield (q/ha) } \\
\hline & 2014 & 2015 & Pooled & 2014 & 2015 & Pooled \\
\hline Weedy check & 34.22 & 34.46 & 34.34 & 40.77 & 41.35 & 41.06 \\
\hline Hand weeding at $30-35$ DAS & 45.81 & 46.99 & 46.40 & 56.06 & 56.33 & 56.20 \\
\hline 2,4-D ester @ $0.5 \mathrm{~kg} / \mathrm{ha}$ at 30 - 35 DAS & 40.73 & 41.56 & 41.15 & 48.80 & 49.04 & 48.92 \\
\hline Sulfosulfuran@25 gm a.i./ha at 30 - 35 DAS & 39.63 & 40.37 & 40.00 & 48.69 & 48.42 & 48.56 \\
\hline Metsulfuran Methyl @ 4 g a.i. / ha at 30 - 35 DAS & 41.38 & 43.58 & 42.48 & 50.07 & 51.16 & 50.61 \\
\hline Sulfosulfuran $75 \%$ +metsulfuran methyl 5\% WG @ 32 g a.i. /ha at 30 - 35 DAS & 43.98 & 45.71 & 44.85 & 53.61 & 55.43 & 54.52 \\
\hline Piroxofop-Propargyl $15 \%$ WP 60 g a.i. /ha at 30 - 35 DAS & 37.03 & 37.56 & 37.30 & 43.87 & 46.09 & 44.98 \\
\hline Clodinafop-propargyl $15 \%$ +metsulfuran methyl $1 \%$ @ 64 g a.i. /ha at 30 - 35 DAS & 44.91 & 46.19 & 45.55 & 54.98 & 55.94 & 55.46 \\
\hline Carfentrazone Ethyl 40 \% DF @ 20 g a.i./ha at 30 - 35 DAS & 42.01 & 44.15 & 43.08 & 50.40 & 51.74 & 51.07 \\
\hline Pendimethalin pre emergence & 38.33 & 39.11 & 38.72 & 46.75 & 48.22 & 47.49 \\
\hline Weed free & 46.75 & 48.69 & 47.72 & 56.17 & 58.40 & 57.29 \\
\hline SEm \pm & 1.40 & 1.60 & 1.06 & 2.03 & 2.34 & 1.55 \\
\hline $\mathrm{CD}(\bar{P}=\mathbf{0 . 0 5})$ & 4.03 & 4.62 & 3.00 & 5.88 & 6.76 & 4.39 \\
\hline
\end{tabular}

Table.4 Residual effect of weed control treatments on germination of succeeding crops

\begin{tabular}{|c|c|c|c|c|c|c|c|c|c|}
\hline \multirow[t]{2}{*}{ Treatments } & \multicolumn{3}{|c|}{$\begin{array}{c}\text { Germination \% } \\
\text { (Pearlmillet) }\end{array}$} & \multicolumn{3}{|c|}{$\begin{array}{l}\text { Germination \% } \\
\text { (Moongbean) }\end{array}$} & \multicolumn{3}{|c|}{$\begin{array}{c}\text { Germination \% } \\
\text { (Clusterbean) }\end{array}$} \\
\hline & 2014 & 2015 & Pooled & 2014 & 2015 & Pooled & 2014 & 2015 & Pooled \\
\hline Weedy check & 94 & 94 & 94 & 94 & 94 & 94 & 94 & 95 & 94 \\
\hline Hand weeding at 30 - 35 DAS & 98 & 98 & 98 & 95 & 97 & 96 & 95 & 95 & 95 \\
\hline 2,4-D ester @ $0.5 \mathrm{~kg} / \mathrm{ha}$ at 30 - 35 DAS & 97 & 96 & 97 & 97 & 97 & 97 & 97 & 97 & 97 \\
\hline Sulfosulfuran@ 25 gm a.i./ha at 30 - 35 DAS & 97 & 97 & 97 & 97 & 97 & 97 & 97 & 97 & 97 \\
\hline Metsulfuran Methyl @ 4 g a.i. / ha at 30 - 35 DAS & 97 & 97 & 97 & 97 & 97 & 97 & 96 & 97 & 96 \\
\hline $\begin{array}{l}\text { Sulfosulfuran } 75 \% \text { +metsulfuran methyl } 5 \% \text { WG @ } 32 \text { g a.i. } \\
\text { /ha at } 30 \text { - 35 DAS }\end{array}$ & 96 & 96 & 96 & 96 & 96 & 96 & 96 & 96 & 96 \\
\hline Piroxofop-Propargyl $15 \%$ WP 60 g a.i. /ha at 30 - 35 DAS & 95 & 95 & 95 & 95 & 95 & 95 & 94 & 95 & 95 \\
\hline $\begin{array}{l}\text { Clodinafop-propargyl } 15 \% \text { +metsulfuran methyl } 1 \% \text { @ } 64 \\
\text { g a.i. /ha at } 30 \text { - 35 DAS }\end{array}$ & 98 & 98 & 98 & 95 & 96 & 96 & 95 & 94 & 95 \\
\hline Carfentrazone Ethyl 40 \% DF @ $20 \mathrm{~g}$ a.i./ha at 30 - 35 DAS & 96 & 96 & 96 & 96 & 97 & 97 & 96 & 96 & 96 \\
\hline Pendimethalin pre emergence & 95 & 95 & 95 & 96 & 97 & 97 & 96 & 96 & 96 \\
\hline Weed free & 98 & 98 & 98 & 94 & 93 & 94 & 96 & 94 & 95 \\
\hline
\end{tabular}


These treatments attained 96 per cent germination. However, the minimum germination per cent was depicted by weed free and weedy check plots i.e. 94 per cent.

\section{Clusterbean}

A perusal of pooled data of two years (Table 4) revealed that highest germination per cent (97\%) was reported for 2, 4-D ester @ 0.5 $\mathrm{kg} / \mathrm{ha}$ and sulfosulfuran @ $25 \mathrm{gm}$ a.i./ha followed by carfentrazone Ethyl 40 \% DF @ $20 \mathrm{~g}$ a.i./ha, pendimethalin pre emergence, metsulfuran methyl @4 g a.i./ ha and sulfosulfuran $75 \%$ +metsulfuran methyl 5\% WG @ $32 \mathrm{~g}$ a.i./ha. These treatments attained 96 per cent germination. However, the lowest germination per cent was depicted by weedy check plots i.e. 94 per cent. These results are in line with those of Yadav et al., (2003), Singh and Ali (2004), Chopra and Chopra (2005), Vala (2005) and Singh et al, (2012).

Based on the results of two years experimentation, it is concluded that conventional method of hand weeding is the most effective and remunerative weed control measure in wheat. Amongst herbicides, clodinafop propargyl $15 \%+$ metsulfuran methyl1\%@64 g a.i /ha or sulfosulfuran 75 $\%+$ metsulfuran methyl 5 WG @ $32 \mathrm{~g}$ a.i./ha found best option for weed control in wheat under especially in labour scarce regions. Further, none of the applied herbicides/mixtures applied in rabi season (wheat) had residual toxicity on predominant crops (pearlmillet, mungbean and clusterbean) grown in kharif season.

\section{References}

Agrawal, K.K. and Jain K.K. 1998. Weed control studies in wheat. World Weeds, 5:69-72.

Bhumesh Kumar, Mishra, J.S., Singh, V.P. and Sharma, A.R. 2016. Challenges of weed management under changing climate. Pp. 203-219. In venkateswalu et al., (Eds) Climate Resilient Agronomy, Indian Society of Agronomy, New Delhi.

Chopra, N. and Chopra, N.K. 2005. Bioefficacy of fenoxaprop, clodinofop, mettribuzin alone and in combination against weed in wheat and their residual effect on succeeding crop. Indian Journal of Weed Science, 37: 163-166.

Gomez, A.A. and Gomez, A.A. 1984. Statistical Procedures for Agricultural Research ( $2^{\text {nd }}$ ed.). John Wiley and Sons. Singapore.

Kumar, J., Kumar, A. and Sharma, B.C. 2010. Effect of chemical and crop establishment methods on weeds and yield of rice and their residual effect on succeeding wheat crop. Indian Journal of Weed Science 42 (1\&2): 78-82.

Nadeem, M.A., Tanveer, A., Ali, A., Ayub, M.K. and Tahir, M. 2007. Effect of weed control practices and irrigation levels on weeds and yield of wheat (Triticum aestivum). Indian Journal of Agronomy52 (1): 60-63.

Sardana, V., Walia, U.S. and Mahajan, G. 2001. Management of broad leaf weeds in wheat (Triticum aestivum L.). Indian Journal of Weed Science33: 69-71.

Sharma, A.R., Bhullar, M.S., Singh, V. Pratap, Singh, Mandeep and Das, T.K. 2016. Harnessing weed-fertilizer-water interactions for higher crop productivity and resource-use efficiency. Indian Journal of Fertilizers, 12(11); 114-130.

Singh, J. and Singh, K.P. 2005. Effect of organic manures on yield and yield attributing characters of wheat. Indian Journal of Agronomy, 50: 289-91.

Singh, P. And Ali, M. 2004. Efficacy of metsulfuron methyl on weeds and its residual effect on succeeding soybean crop grown on vertisols of Rajasthan. Indian Journal of Weed Science36: 34- 
37.

Singh, R., Shyam, R., Singh, V.K., Kumar, J., Yadav, S.S. and Rathi, S.K. 2012. Evaluation of bioefficacy of clodinafoppropargyl + metsulfuron-methyl against weeds in wheat. Indian Journal Weed Science, 44(2): pp 81-83.

Surin, S.S., Singh, M.K., Upasani, R.R., Thakur, R. and Pal, S.K. 2013. Weed management in rice (Oryza sativa)wheat (Triticum aestivum) cropping system under conservation tillage. Indian Journal of Agronomy 58(3): 288291.
Yadav,A., Mehta, R., Punia, S. S.,Hooda, V., Malik, R. R., Rana, V. and Brllinder, R. R. 2003 Resudual effect of four sulfonylurea herbicides applied on wheat on succeeding crops in rotation. Indian Journal of Weed Science 35: 259-261.

Vala, G.R. 2005. Efficacy of various herbicides and determination of their persistence through bioassay technique for summer Groundnut (Arachis hypogaea L.). Ph.D. (Agri.) thesis submitted to Junagadh Agricultural University, Junagadh (Gujarat).

\section{How to cite this article:}

Yadav, H.L., A.K. Gupta, Sudesh Kumar and Shweta Gupta. 2019. Comparative Efficacy of Herbicides Applied in Wheat and their Residual Effect on the Succeeding Crops. Int.J.Curr.Microbiol.App.Sci. 8(04): 1866-1874. doi: https://doi.org/10.20546/ijcmas.2019.804.218 